\title{
Las Tecnologías de la Información y la Comunicación en las radios universitarias españolas en el periodo 2012-2016
}

\author{
Information and Communications Technologies \\ in the Spanish university radios in \\ the period 2012-2016
}

\author{
Daniel MARTÍN-PENA ${ }^{1}$ \\ Macarena PAREJO-CUELLAR ${ }^{2}$ \\ Agustín VIVAS-MORENO ${ }^{3}$
}

\section{Resumen}

Las Tecnologías de la Información y la Comunicación han posibilitado el paso de una radio tradicional a otra bidireccional, colaborativa, democrática y de grandes posibilidades de alcance. El presente artículo tiene por objetivo analizar la evolución de las estaciones universitarias españolas a través de las Tecnologías de la Información y la Comunicación en los últimos cinco años. Los criterios metodológicos seguidos han sido: la entrevista para obtener la información necesaria, la elaboración de un instrumento de análisis, la recolección de datos a través de la observación y exploración directa y el análisis comparativo de los mismos. Se canalizan los resultados en torno a tres cuestiones: la emisión, la interactividad y el cambio y adaptación de la Web. Se concluye que, a pesar de que las Tecnologías de la Información y la Comunicación han contribuido a la supervivencia y extensión del fenómeno radiofónico universitario en España en el último lustro, no se están aprovechando todas las potencialidades que se derivan de su extensión en un entorno 3.0.

Palabras clave: Emisión. Interactividad. Radio. Tecnologías de la información y la comunicación. Universidad.

\begin{abstract}
Information and Communications Technology has enabled the transition from a traditional two-way radio to another, democratic and collaborative, with greater reach possibilities. This study aims to analyze the evolution of the Spanish university stations through Information and Communications Technologies in the past five years. The methodological approaches pursued were: the interview as a means to obtain the necessary information, the development of an analytical tool, collecting data through observation and direct exploration, and comparative analysis of the data. The results are analyzed focusing on three issues: broadcasting, interactivity, and switch and adaptation to the Web. In conclusion, Information and Communications Technology has meant the survival of the radio phenomenon in Spain in the last five years. In fact, one can see a continuous evolution of the station universities, althouth it is not always positive, since they have not taken up every possible potential that Information and Communications Technology has in the environment 3.0 .
\end{abstract}

Keywords: Broadcasting. Interactivity. Radio. Information and communications technology. University.

1 Universidad de Extremadura, Facultad de Ciencias de la Documentación y la Comunicación, Departamento de Información y Comunicación. Plazuela Ibn Marwan, s/n., 06001, Badajoz, España. Correspondencia a nombre de/Correspondence to: D. MARTíN-PENA. E-mail:<danielmartin@unex.es>.

2 Universidad de Extremadura, Difusión de la Cultura Científica. Badajoz, España.

3 Universidad de Extremadura, Instituto de Lenguas Modernas, Departamento de Información y Comunicación. Badajoz, España. Recibido el 19 del diciembre del 2016, versión final re-presentada el 4 del mayo del 2017 y aprobado el 31 del mayo del 2017.

Como citar este artigo/How to cite this article

Martín-Pena, D.; Parejo-Cuellar, M.; Vivas-Moreno, A. Las tecnologia de la información y la comunicación en las radios universitarias españolas en el periodo 2012-2016. Transinformação, v. 30, n. 1, p. 27-38, 2018. https://doi.org/10.1590/2318-08892018000100003 


\section{Introducción}

La comunicación siempre ha estado vinculada a los avances tecnológicos (Rodríguez-Pallarés; Rodríguez Barba; Peinado, 2016). Las Tecnologías de la Información y la Comunicación (TIC), lejos de catapultar la radio, la han trasformado en un nuevo medio, donde las formas innovadoras de distribuir contenidos se conjugan con los formatos tradicionales (Alonso González, 2015). En ese estado se encuentra la radio universitaria, piedra angular de la presente investigación que tiene por objeto analizar cómo han evolucionado desde el punto de vista tecnológico en los últimos años (2012-2016).

Antes de exponer los principios metodológicos y los resultados, resulta conveniente aproximarnos al modelo de convergencia tecnológica de la radio universitaria en España.

\section{Radio y Tecnologías de la Información y la Comunicación: convergencia tecnológica}

Si algo hubiese que anotar sobre la larga trayectoria de la radio sería que, en su devenir, ha venido caminando hacia un modelo más accesible, más autónomo (López Vidales, 2011) y con novedosos formatos (Ortiz-Sobrino, 2011). Una situación favorecida por el paso a la Web semántica o Web 3.0. Y es que como subrayan Küster y Hernández (2013, p.106) este entorno "tiene la capacidad de construir un cúmulo de conocimientos sobre las preferencias de los usuarios capaz de atender de forma exacta las demandas".

A pesar de ello, no pensemos que las sustanciales transformaciones hayan redefinido un nuevo medio (post-radio). Como opinan Ortiz-Sobrino (2012) y Ramos Ruiz (2015), los nuevos escenarios acaban adaptándose al entorno y el paso de la radio hertziana a la de internet ha supuesto una evolución que no ha hecho tabla rasa del pasado.

Lo que sí ha supuesto la red ha sido una metamorfosis. Veamos, sin ánimo de exhaustividad, algunas de las características que definen la radio en esa inquebrantable innovación:

a) Radio bidireccional: El medio ofrece grandes posibilidades de retroalimentación, haciendo parte del proceso comunicativo al oyente (Piñeiro-Otero; Videla Rodríguez, 2013).

b) Radio colaborativa: Adquiere distintas formas de llegar con su mensaje al público, desde emisión en directo hasta ofrecimiento de enlaces para recuperar contenido (Barrios, 2013). No hay fronteras, el oyente escucha cuando y donde quiere (Padilla; Calvo, 2011). Existe la posibilidad de aumentar, completar, actualizar y compartir los contenidos e interactuar con el usuario a través de nuevas plataformas (Ventero; Peña, 2011).

c) Radio transnacional: Internet ha roto las barreras y ha generado diferentes modalidades que van desde la radio creada exclusivamente para la red hasta la convencional por ondas. Existe así una variada tipología en un contexto donde adquieren protagonismo las pantallas y la telefonía, ampliando el universo de difusión (PiñeiroOtero, 2015a, 2015b).

d) Radio democrática: La variedad programática ha dibujado una nueva audiencia (Ramos Ruiz, 2015). El usuario, cuando se asoma a la red, encuentra ventajas como una radio ajustada a los gustos de la audiencia, un diálogo más cercano o una ventana a infinidad de contenidos y formatos.

e) Radio de grandes potencialidades: El podscasting, la interactividad y la gestión documental son algunos elementos por reseñar. El podscasting ha sido estudiado por diversos autores (Cebrián, 2008; Gallego, 2010). Aguayo (2015) lo define como un contenido que puede descargarse, generado automáticamente y que puede ser escuchado en cualquier momento y lugar. Con internet, la audiencia puede crear una parrilla de programas a la carta (Pérez Alaejos; López Merayo, 2013). El desafío sigue siendo la interactividad, como concepto que integra la hipertextualidad y la multimedialidad (Díaz-Noci, 2010). Por último, la gestión documental como mecanismo clave 
para el desarrollo del futuro radiofónico en la búsqueda y recuperación de la información (Marta-Lazo; Ortiz-Sobrino, 2013).

Al final, la radio adquiere un carácter "glocal" donde el contenido que se genera en el municipio puede tener un alcance global. No se debe obviar que la proximidad al radioescucha ha sido un principio del medio radiofónico desde sus orígenes (Terol, 2016).

\section{Evolución: emisoras universitarias en España}

Son estaciones jóvenes, de apenas 40 años de recorrido, con un desarrollo similar al de otros países europeos, pero muy diferente del contexto americano, en el que países como Argentina o Estados Unidos han abanderado esta radiodifusión desde los años 20 (Aguaded; Martín-Pena, 2013). Marta-Lazo y Martín-Pena (2014) señalan que la evolución española puede delimitarse en tres fases:

a) Una etapa inicial con acercamientos de la universidad a las radios convencionales (Ortiz-Sobrino; Peña, 2010). El inicio de las transmisiones universitarias lo protagoniza Radio Uned en 1974. Esta fase está marcada por un desarrollo lento. Surgen una decena de estaciones (Martín-Pena; Contreras, 2014).

b) Etapa de expansión (primera década del Siglo XXI). Surge una quincena de emisoras en marcha de talleres de radio y aprovechamiento TIC (Martín-Pena; Contreras; Aguaded, 2015). En este aspecto, tal y como afirman Espino y Martín-Pena (2012, p.22), gracias a la explotación de las TIC, "la aventura de montar una emisora universitaria se torna una tarea al alcance de cualquier universidad".

c) La tercera fase (últimos seis años) en la que se experimenta una contención en la evolución vivida.

Sin duda, las TIC han tenido un papel destacado en el desarrollo de las emisoras universitarias españolas (Martín-Pena; Parejo; Vivas, 2016). No en vano, Internet ha facilitado la aparición de emisoras universitarias (Gallego, 2011; Perona Paéz, 2009) y ha permitido desarrollar "una producción muy alejada de los costes de emisión y limitación de frecuencias" (Marta-Lazo; Segura, 2011, p.327).

\section{Procedimientos metodológicos}

El objetivo ha sido analizar la evolución de las estaciones universitarias españolas a través de las TIC en los últimos cinco años. A partir de este propósito se han establecido unos objetivos específicos:

a) O1: Identificar criterios e indicadores para el estudio de las TIC en las radios universitarias.

b) O2: Analizar la evolución en los últimos cinco años de los indicadores TIC extraídos.

c) O3: Estudiar la evolución del fenómeno radiofónico universitario, examinando de qué forma las TIC han contribuido a la creación de las nuevas radios.

Para ello, se ha partido de tres hipótesis de trabajo:

a) H1: Existe una clara relación entre el desarrollo de las TIC y el actual tejido radiodifusor universitario español.

b) H2: La intensidad en el uso de las TIC en las radios universitarias ha ido incrementándose en el mismo nivel que lo ha hecho la Web 3.0.

c) H3: La interactividad sigue siendo una asignatura pendiente para las radios universitarias españolas.

Para la selección de la muestra se han tomado como base las emisoras universitarias insertas en la Asociación de las Radios Universitarias de España (ARU). Nuestro estudio se encuadra en una línea de trabajo del Grupo de Investigación ARDOPA (Grupo Gestión de la Información, la Documentación y el Patrimonio en las Organizaciones) en la Universidad de Extremadura. Es un análisis evolutivo con dos fechas de referencia. Por un lado, la temporada 
2012/2013 (periodo 1), y por otro, la 2015/2016 (periodo 2). En el periodo 1, la ARU estaba compuesta por 24 emisoras y en el 2, agrupaba a 25. Por tanto, conviene anotar las siguientes apreciaciones:

a) Las emisoras que se han mantenido invariables en ambos periodos han sido un total de 22, y están asociadas con código numérico correlativo:

- Radio Uned: 1.

- Radio Campus: 2.

- InfoRadio: 3.

- Radio Universidad Salamanca: 4.

- Radio Universidad Navarra: 5.

- Radio Universitaria León: 6.

- Europea Radio: 7.

- UPV Ràdio: 8.

- VoxUJI Ràdio: 9.

- Onda Campus: 10.

- Radio CEU: 11.
- UniRadio Huelva: 12.

- UPF Ràdio: 13.

- Radio UMH: 14.

- RUAH Alcalá: 15.

- Radio URJC: 16.

- iRadio Ucam: 17.

- UAL Radio: 18.

- UniRadio Jaén: 19.

- OnCEULab: 20.

- Radio.UniZar.es: 21.

- Radio USJ: 22.

b) Las emisoras Radio SIDE (código A) y Radio Universitat (código B) han dejado de emitir y figuran sólo en el periodo de análisis 1 .

c) Las emisoras Radio UVa (código C), Radio Politécnica (código D) y UAB Ràdio (código E) se han integrado a la ARU recientemente. Figuran en el periodo 2.

Estas oscilaciones han sido tomadas en cuenta para establecer los porcentajes.

El proceso metodológico se ha desarrollado en las siguientes fases:

a) Acceso a campo de investigación a través de la entrevista telefónica semiestructurada: Ha sido el mecanismo utilizado para obtener la información, los criterios e indicadores:

- Los planteamientos fueron realizados a través de preguntas dicotómicas (mucho/normal/poco), con posibilidad de explicación de la respuesta.

- Éstas fueron revisadas previamente por un comité de expertos (profesores universitarios y dirigentes de radios) a través de un cuestionario de validación.

- Tratamiento de las respuestas obtenidas con Nvivo 9.

- Segunda ronda con los responsables de las emisoras para refrendar el modelo.

Las preguntas de las entrevistas constituyen el Cuestionario 1 y son:

- ¿En qué aspectos influyen las TIC en las emisoras universitarias?

- ¿Qué importancia tienen las TIC en estas emisoras?

- ¿Qué importancia tiene la interactividad en tu emisora?

- ¿Ha mejorado la conexión con tus usuarios en los últimos años?

- ¿Qué importancia tiene el tener presencia en nuevas pantallas: smartphones y tablets?

- ¿Qué importancia tiene el acceso a la radio a la carta?

b) La siguiente fase corresponde a la elaboración de un instrumento de análisis basado en criterios e indicadores obtenidos: 
- Los criterios que obtuvieron consenso en la segunda ronda de contactos con los responsables pasaron a formar parte del instrumento diseñado (Cuadro 1). Un cuestionario compuesto por tres criterios genéricos y siete específicos, y sus indicadores (31), que se han utilizado para evaluar la muestra del estudio. Dicho instrumento queda configurado en tres bloques: emisión, interactividad y plataforma Web.

c) En la tercera fase se realiza la recolección de datos, aplicando la herramienta diseñada, a través de la exploración online de cada emisora: diseño de un procedimiento para los aspectos genéricos de emisión; Recolección de los datos de las radios como unidades de análisis independientes, y tratamiento de la información, para valorar el cumplimiento o no, con SPSS Inc.

d) Análisis comparativo.

Cuadro 1. Instrumento de análisis TIC.

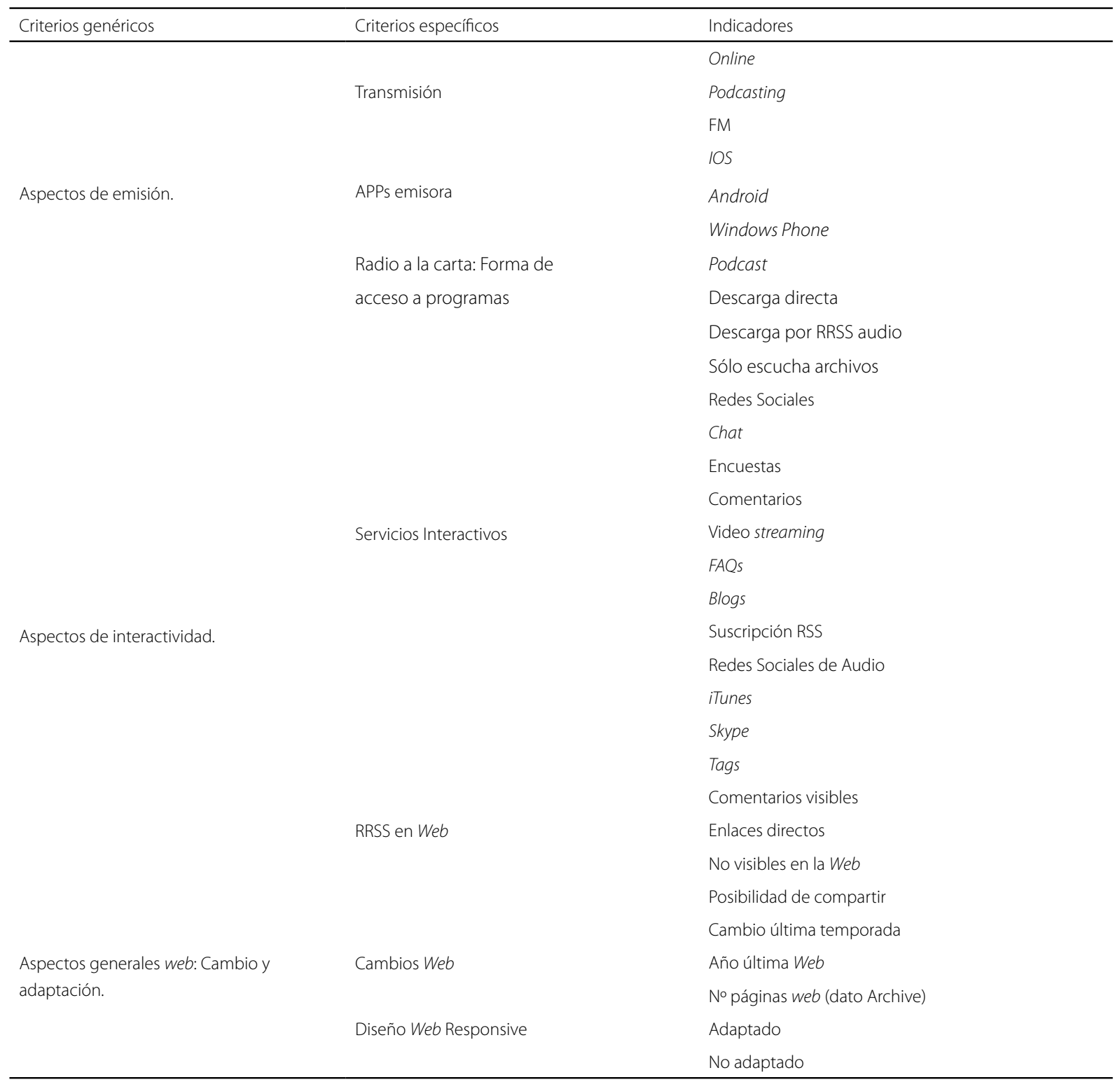




\section{Resultados y Discusión}

Mediante el proceso metodológico se han obtenido resultados que permiten analizar, comparar y evaluar la implementación de las TIC y las diferencias globales entre las distintas radios universitarias.

\section{Aspectos relacionados con la emisión}

a) Transmisión: la emisión a través FM y la transmisión online (streaming) - Tabla 1 - son las fórmulas primarias más usadas por estas emisoras, el podcast como forma de emisión exclusiva queda por detrás, aunque se ha constatado que todas las radios del estudio realizan en la actualidad subida de archivos y un $72 \%$ hace streaming de su programación. Hay pequeñas diferencias entre ambos periodos (Tablas 1 y 2): el porcentaje de emisoras que emiten en FM se ha estancado y el streaming va en claro ascenso (siete puntos porcentuales). Se constata un mínimo retroceso de la radio tradicional y un avance de la radio por red. Es habitual que las radios universitarias arranquen sus emisiones a través de la subida de archivos, pero con el tiempo evolucionan a una programación 24 horas, normalmente vía streaming.

Tabla 1. Aspectos generales emisión (Temporada 2015/2016).

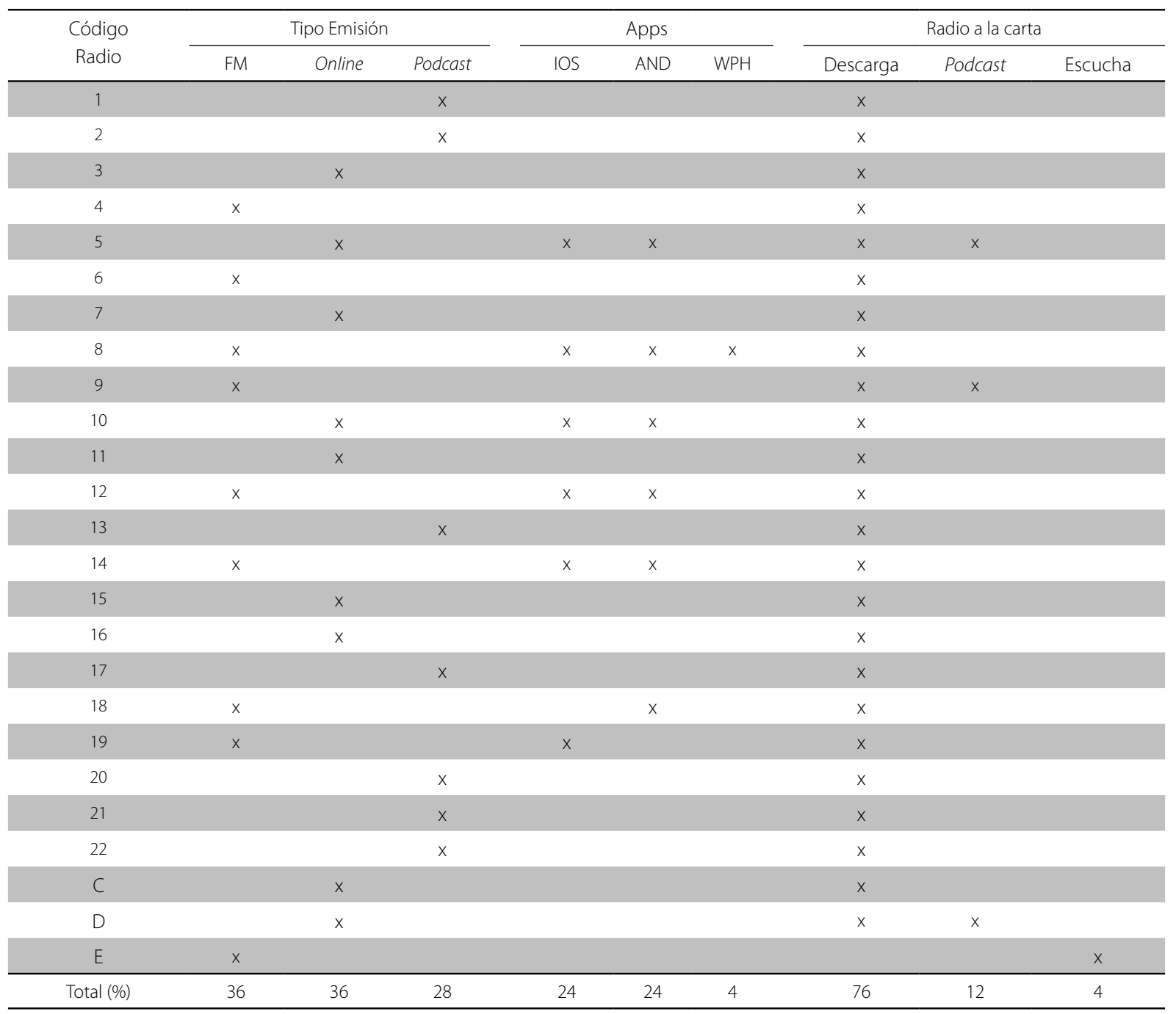

Fuente: Elaborado por el autor (2016). 
b) APPs (Applications): el desarrollo de esta tecnología es significativo, teniendo en cuenta el público objetivo de este tipo de emisoras, nativos digitales (Benavides, 2012). En estas estaciones la implementación de esta tecnología está siendo muy lenta, sin incidencia y evolución. Es significativo que, desde el periodo de análisis inicial, el número de radios universitarias que desarrollan aplicaciones para Smartphones ha doblado, aunque sigue siendo un porcentaje bajo. Tan sólo un 24\% de las emisoras analizadas poseen APPs bajo sistemas IOS y Android (Radio Universidad Navarra, OndaCampus, Radio UPV, Radio UMH, UniRadio Huelva y Jaén). Las aplicaciones desarrolladas bajo el sistema operativo Windows Phone únicamente aparecen en el segundo periodo de análisis (2015/2016) y tan solo está presente en Radio UPV. La mayor parte de las aplicaciones no son propias de las emisoras, se encuentran integradas en la APP de la institución correspondiente. La única emisora que tiene desarrolladas APPs bajo los tres sistemas es Radio UPV.

c) Radio a la carta: otro criterio específico es el acceso a la escucha/descarga de programas. La penetración del podcasting es del 100\%. Las emisoras ofrecen el servicio de radio a la carta a través de tres fórmulas:

- Escucha o visualización de audios: es minoritario y se da sólo en UAB Ràdio.

- Suscripción podcast: estos servicios han descendido ligeramente pasando de cuatro a tres emisoras (VoxUJIRàdio, Radio Universidad de Navarra y Radio Politècnica).

Tabla 2. Aspectos generales emisión (Temporada 2012/2013).

\begin{tabular}{|c|c|c|c|c|c|c|c|c|}
\hline \multirow{2}{*}{$\begin{array}{c}\text { Código } \\
\text { Radio }\end{array}$} & \multicolumn{3}{|c|}{ Tipo Emisión } & \multicolumn{2}{|c|}{ Apps } & \multicolumn{3}{|c|}{ Radio a la carta } \\
\hline & FM & Online & Podcast & IOS & Android & Descarga & Podcast & Escucha \\
\hline 1 & & & $x$ & & & $x$ & & \\
\hline 2 & $x$ & & & & & & & \\
\hline 3 & & $x$ & & & & $x$ & & \\
\hline 4 & $x$ & & & & & $x$ & & \\
\hline 5 & $x$ & & & $x$ & $x$ & $x$ & $x$ & \\
\hline 6 & $x$ & & & & & & & \\
\hline 7 & & $x$ & & & & $x$ & & \\
\hline 8 & $x$ & & & & & $x$ & & \\
\hline 9 & $x$ & & & & & $x$ & $x$ & \\
\hline 11 & & $x$ & & & & $x$ & & \\
\hline 12 & $x$ & & & $x$ & & $x$ & $x$ & \\
\hline 13 & & & $x$ & & & $x$ & $x$ & \\
\hline 14 & $x$ & & & & & $x$ & & \\
\hline 15 & & $x$ & & & & $x$ & & \\
\hline 16 & & $x$ & & & & & & $x$ \\
\hline 17 & & & $x$ & & & $x$ & & \\
\hline 18 & & & $x$ & & $x$ & $x$ & & \\
\hline 19 & $x$ & & & & & $x$ & & \\
\hline 20 & & & $x$ & & & $x$ & & \\
\hline A & & & $x$ & & & & & \\
\hline B & & $x$ & & & & $x$ & & \\
\hline Total (\%) & 37 & 29 & 33 & 12 & 12 & 79 & 17 & 4 \\
\hline
\end{tabular}

Fuente: Elaborado por el autor (2016). 
- La descarga directa es mayoritaria. Se localizan dos alternativas: el desarrollo del servicio de descarga propio a través de la puesta en marcha de la subida de audios (en claro descenso, de un $46 \%$ a un 36\%), o, a través de la utilización de las redes sociales de audio (opción ascendente y más operativa para estas radios, un aumento del 14\%). En el periodo de análisis 1, las dos opciones estaban prácticamente igualadas (diferencia del 4\%). En el periodo 2, la diferencia se eleva al 28\% a favor de la utilización de servidores externos.

\section{Aspectos de interactividad en la Web}

La radio de hoy se perfila como un medio mucho más cercano al oyente, con mayores dosis de interactividad. Como indica Terol (2016), el receptor se convierte en prosumidor de contenidos y participa del mensaje. La interactividad es para las radios universitarias una herramienta indispensable con la cual lograr establecer ese diálogo con los oyentes. Dada su importancia, se ha hecho especial hincapié en observar cómo utilizan esta herramienta las radios universitarias:

a)Servicios interactivos: la radio siempre ha intentado dar respuesta al oyente, pero nunca ha gozado de tantas opciones (Figura 1):

- Los servicios interactivos de mayor penetración en 2012/2013 (redes sociales, redes sociales de audio y suscripción RSS) continúan ocupando esas posiciones, pero con mayores índices de penetración, a excepción de la suscripción RSS que desciende.

- Las redes sociales tienen una presencia sistémica. Twitter con una cota del 100\% y Facebook del 84\% despuntan sobre otras (Youtube, Google, Instagram, Flickro Tumblr). La presencia de Tuenti, que se daba en 3 emisoras

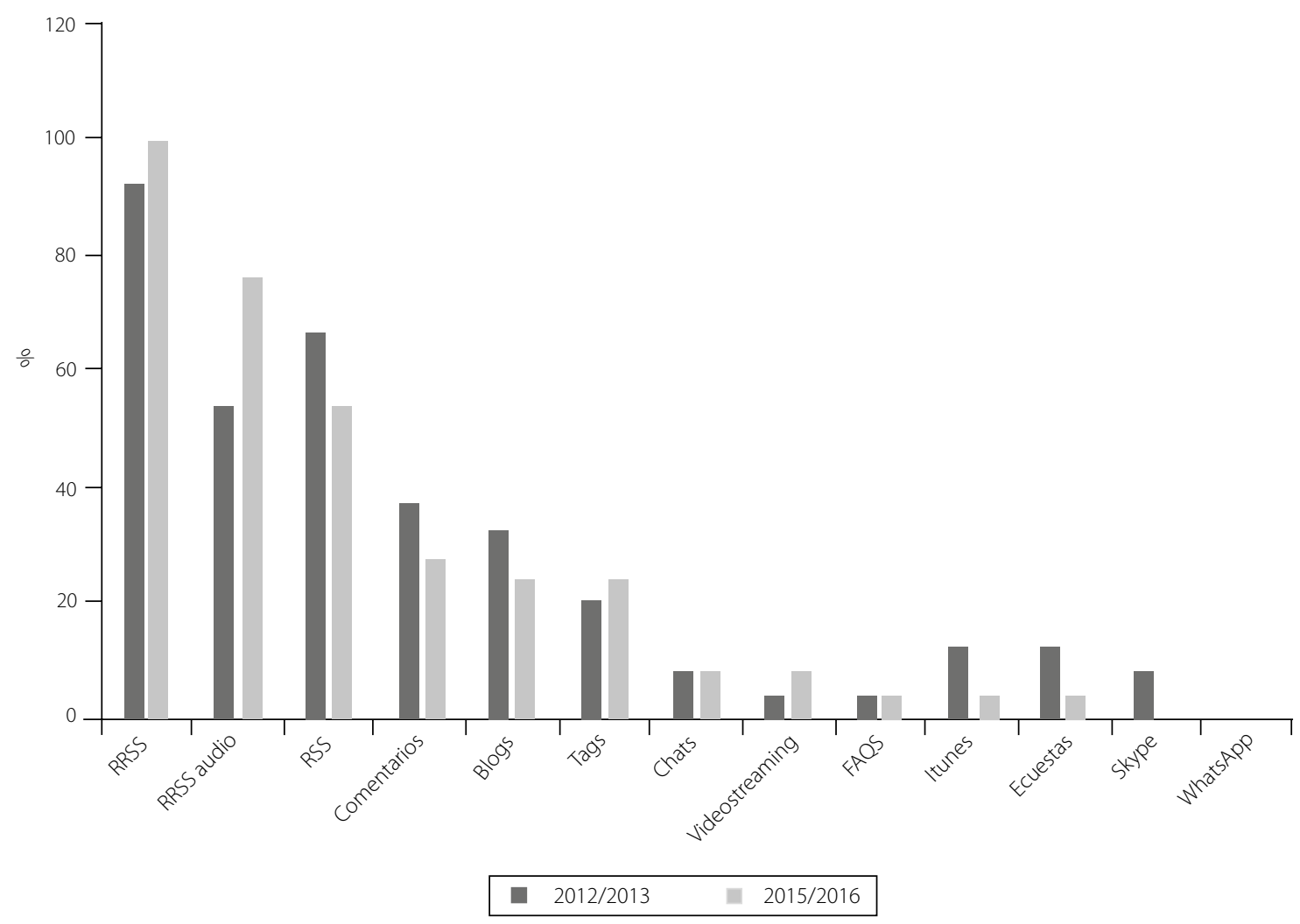

Figura 1. Servicios interactivos.

Fuente: Elaborado por el autor (2016). 
en 2012/2013, ha desaparecido. En segunda posición se encuentran las redes sociales de audio (iVoox, SoundCloud o uWhish) que han sufrido el mayor porcentaje de incremento (46\%). La mayor presencia se detecta en iVoox con un 68\%, las otras dos redes quedan con valores inferiores al $8 \%$.

- Las tags y el videostreaming son otros dos servicios que aumentan su presencia.

- El resto de servicios sufre descensos. Tanto Skype como WhastApp no tienen presencia en la web.

b) Presencia de redes sociales en la Web: son el servicio interactivo hegemónico (Figura1):

- Enlaces directos a los perfiles sociales: ha aumentado. Es el más usado (de 13 a 17 emisoras).

- Comentarios visibles de los seguidores: han pasado a ocupar la segunda posición con la mayor tasa de incremento, de 8 a 13 emisoras. Son más visibles los comentarios de Twitter (12 emisoras) que Facebook (3 radios).

- Posibilidad de compartir audios en las distintas redes sociales: los valores se mantienen, un total de 12 emisoras.

Tabla 3. Aspectos Generales Web (2015/2016).

\begin{tabular}{|c|c|c|c|c|c|c|}
\hline \multirow{2}{*}{$\begin{array}{l}\text { Código } \\
\text { Radio }\end{array}$} & \multicolumn{4}{|c|}{ Cambio y No Webs } & \multicolumn{2}{|c|}{ Disegn Web Responsive } \\
\hline & sí & $\mathrm{NO}$ & No & Año Web & sí & $\mathrm{NO}$ \\
\hline 1 & & $x$ & $S / R$ & 2013 & & $x$ \\
\hline 2 & & $x$ & $S / R$ & 2006 & & $x$ \\
\hline 3 & $x$ & & 6 & 2016 & $x$ & \\
\hline 5 & & $x$ & 4 & 2013 & & $x$ \\
\hline 6 & & $x$ & 3 & 2013 & & $x$ \\
\hline 7 & $x$ & & 6 & 2016 & $x$ & \\
\hline 8 & $x$ & & 4 & 2015 & & $x$ \\
\hline 10 & & $x$ & 3 & 2011 & & $x$ \\
\hline 11 & $x$ & & 2 & 2015 & & $x$ \\
\hline 12 & $x$ & & 4 & 2016 & $x$ & \\
\hline 13 & $x$ & & 3 & 2015 & $x$ & \\
\hline 14 & & $x$ & 2 & 2012 & & $x$ \\
\hline 15 & & $x$ & 3 & 2012 & $x$ & \\
\hline 16 & $x$ & & 2 & 2015 & $x$ & \\
\hline 17 & $x$ & & 4 & 2015 & $x$ & \\
\hline 18 & & $x$ & 3 & 2014 & $x$ & \\
\hline 19 & & $x$ & 4 & 2011 & & $x$ \\
\hline 21 & & $x$ & 1 & 2011 & & $x$ \\
\hline 22 & $x$ & & 3 & 2015 & $x$ & \\
\hline$C$ & & $x$ & 1 & 2014 & & $x$ \\
\hline $\mathrm{D}$ & & $x$ & 1 & 2011 & & $x$ \\
\hline$E$ & $x$ & & 1 & 2015 & $x$ & \\
\hline Total (\%) & 48 & 52 & & & 48 & 52 \\
\hline
\end{tabular}

Fuente: Elaborado por el autor (2016). 
- Un problema detectado en 2012/2013, y que aún se mantiene en dos emisoras, es que las redes sociales no tienen su espacio en los portales de las emisoras (Radio Campus y Radio Universitaria León).

\section{Aspectos generales en las Webs: cambios y adaptación}

También se han analizado otros aspectos generales que influyen en la forma en la que la radio proyecta sus mensajes e interactúa con el público.

a) Cambio de Web.

Uno de los criterios genéricos a observar es cómo evolucionan las webs de las emisoras y si lo hacen de manera paralela al desarrollo tecnológico. A través de la plataforma Archive.org ha sido posible acceder a una librería-archivo donde se almacena un registro de la apariencia de las Webs en sus distintos años. En la Tabla 4 se observa que la mitad (48\%) de las emisoras han cambiado sus plataformas en la última temporada (2015/2016). Por el contrario, son tres las radios que tienen plataformas con más de 6 años. Es el caso de la veterana Radio Campus.

Otro de los indicadores evaluados es el número de veces que las emisoras han renovado sus Webs desde que existen registros. El fenómeno radiofónico universitario español es relativamente joven, dado que desde 2007 han aparecido 14 de las 25 emisoras analizadas. Un 56\% de las radios tienen menos de la decena de años y han surgido ya en un entorno con un desarrollo TIC. Según la Tabla 3 un total de 11 emisoras han cambiado entre 3 y 4 veces de plataforma Web desde su existencia, y otras 9 radios lo han hecho en 1 o 2 ocasiones. Menos habitual, y para emisoras más veteranas (más de 15 años de trayectoria), se han producido 5 o más cambios (InfoRadio, Radio Universidad Salamanca o Europea Radio). Estos cambios suelen adaptar las tecnologías existentes en cada momento, aunque no siempre sucede así, y son pocas las emisoras universitarias que plantean plataformas adaptadas a la web semántica.

b) Diseño Web adaptativo.

Como afirman Gonzálezy Marcos (2013, p.1)"el aumento de los teléfonos móviles y tabletas lleva a plantearse un diseño adaptado a estos aparatos". El 48\% de las emisoras analizadas en la temporada 2015/2016 cuentan con un Diseño Web Responsive. El resto de radios son meros traslados de su versión Web a los nuevos dispositivos inteligentes.

\section{Conclusión}

A la luz de los resultados obtenidos se pueden establecer las siguientes conclusiones:

1) Las TIC han supuesto la supervivencia del fenómeno radiofónico en España en el último lustro. Se apuesta por el medio universitario totalmente online y que poco a poco se aleja de los cánones tradicionales de radio. El abaratamiento de los costes, fundamental en un contexto universitario delicado, junto a las dificultades legales de acceso a licencias, quizás sean los motivos que lo justifiquen y que hacen que las emisoras universitarias apuesten por las nuevas formas de emisión derivadas de las TIC.

2) Se constata una evolución continua de las estaciones universitarias, aunque no siempre positiva:

- En la emisión se observa el avance de la radio por red y la penetración de la radio a la carta y el podcast. Otros recursos resaltan por su casi total ausencia, tales como las APPs propias de las emisoras; la falta de recursos para su desarrollo puede ser el motivo, por ello se deberían establecer sinergias con investigadores propios de la universidad, que sean expertos en estas áreas del conocimiento, para lograr implementar esta tecnología al menor costo posible, ya que la situación económica de las radios es la que no permite el desarrollo de estas aplicaciones y no tanto la falta de interés en las mismas. 
- La interactividad en los medios es consecuencia directa de la implementación de las TIC. Las redes sociales son el servicio interactivo hegemónico, además de las ya tradicionales (Twittery Facebook, por ese orden), fundamentalmente las vinculadas al audio (iVoox) han supuesto un cierto alivio para estas emisoras, tanto para la mejora del posicionamiento de los programas como para el ahorro que supone no tener que desarrollar servicios propios de subida y bajada de audios, que puedan saturarse de manera habitual. El resto de los servicios interactivos sufre un descenso preocupante por su falta de aprovechamiento. No nos cabe duda de que la tendencia actual apunta a que aplicaciones como WhatsApp tendrán en próximos estudios de estas emisoras una penetración mayor, por la facilidad de uso y las posibilidades interactivas que puede brindar y que ya están siendo testadas por emisoras convencionales.

- Se verifica la constante transformación en la que se encuentran los portales, tanto en lo que respecta a su estructura como a la prestación de servicios, incrementándose el Diseño Web Responsive. Aunque se ha de apuntar que muchas emisoras adolecen de personal experto en programación web y se limitan al desarrollo de plataformas web basadas en tecnologías como Wordpress y otros sistemas que no aprovechan todas las oportunidades actuales.

3) Si bien la radio universitaria en la era digital ha llevado al desarrollo de experiencias globales de comunicación, se constata que no se han aprovechado todas las potencialidades TIC. Queda por avanzar en nuevas técnicas y nuevos servicios para usuarios. El marco universitario es propicio para ello, para innovar y proponer mejoras en el ámbito de la emisión, la participación, la interactividad o los formatos, pero se ha detectado que la situación económica de las emisoras no es, actualmente, la propicia.

A través de todos estos datos se puede comprobar cómo las hipótesis iniciales de partida se cumplen de manera parcial:

- H1: queda demostrada, dado que hay una clara relación entre el desarrollo de las TIC y el actual tejido radiodifusor español y las nuevas radios universitarias que van surgiendo. Se ha podido observar que las emisoras que surgen lo hacen en formato online o a la carta.

- H2: ha sido refutada, dado que en la mayoría de las estaciones los servicios ofrecidos son sustitutivos y no complementarios. En consecuencia, el uso de las TIC no ha ido incrementándose al nivel de la Web 3.0.

- H3: queda demostrada, dado que muchos recursos de interactividad están en retroceso, y sigue siendo una asignatura pendiente para las radios universitarias españolas, que se centran casi exclusivamente en los medios sociales sin aprovechar la infinidad de recursos existentes.

Este trabajo deja la puerta abierta a futuras investigaciones. Los datos podrían ser ponderados en un posterior estudio para la confección de un ranking que permitiera dilucidar qué emisoras son las que han implementado los recursos digitales. Tampoco debe olvidarse, sobre todo si se tiene en cuenta la enorme obsolescencia que caracteriza el contexto de las radios universitarias, que los resultados extraídos suponen un valioso material documental para establecer nuevas comparativas que permitan evaluar el desarrollo futuro.

\section{Colaboración}

D. Martín-Pena contribuye a supervisión, acceso a campo, resultados y conclusiones. M. Parejo-Cuellar contribuye a repaso marco teórico, discusión y conclusiones. A. Vivas-Moreno contribuye a preparación, supervisión de la metodología de la investigación y conclusiones.

\section{Referencias}

Aguaded, J. I.; Martín-Pena, D. Educomunicación y radios universitarias: panorama internacional y perspectivas futuras. Chasqui, n. 124, p. 63-70, 2013. 
Alonso González, M. A Radio y Redes sociales: la interactividad radiofónica en los 'Morning Shows'en España. Estudios sobre el Mensaje Periodístico, v. 21, n. 2, p. 689-704, 2015.

Barrios, A. La radio en la era de la sociedad digital. adComunica: Revista Científica Estrategias, Tendencias e Innovación en Comunicación, n. 5, p. 39-54, 2013.

Benavides, E. El sistema operativo Android en la radio universitaria: la implicación de los estudiantes. In: Espino, C.; Martín-Pena, D. (Ed.). Las radios universitarias, más allá de la radio: las TIC como recursos de interacción radiofónica. Barcelona: UOC, 2012. p. 245-260.

Cebrián, M. La radio en Internet: de la ciberradio a las redes sociales y a la radio móvil. Buenos Aires: La Crujía, 2008

Díaz-Noci, J. Medios de comunicación en internet: algunas tendencias. El Profesional de la Información, v. 19, n. 6, p. 561-567, 2010.

Espino, C.; Martín-Pena, D. (Ed.). Las radios universitarias, más allá de la radio: las TIC como recursos de interacción radiofónica. Barcelona: UOC, 2012.

Gallego, J. I. Podcasting: Distribución de contenidos sonoros y nuevas formas de negocio: la empresa radiofónica española. 2009. 487 f. Tesis (doctoral) - Universidad Complutense, Madrid, 2010.

Gallego, J. I. Alternativas e innovación en las radios universitarias españolas. In: Aguaded, J. I; Contreras, P. (Coord.). La radio universitaria como servicio público para una ciudadanía democrática. A Coruña: Netbiblo, 2011. p. 97-108.

González, D.; Marcos, M. C. Responsive web design: diseño multidispositivo para mejorar la experiencia de usuario. BiD: Textos Universitaris de Biblioteconomia i Documentació, v. 8, n. 31, p.1-10. 2013.

Küster, I.; Hernández, A. De la Web 2.0 a la Web 3.0: antecedentes y consecuencias de la actitud e intención de uso de las redes sociales en la web semántica. Universia Business Review, n. 37 , p. 104-119, 2013.

López Vidales, N. La radio se transforma: nuevas tecnologías, nuevos hábitos y nuevos perfiles para el medio más cercano. In: Ortiz Sobrino, M. A.; López Vidales, N. (Ed.). Radio 3.0: una nueva radio para una nueva era. La democratización de los contenidos. Madrid: Fragua. 2011. p. 15-40.

Marta-Lazo, C.; Martín-Pena, D. Investigación sobre radio universitaria: presente, pasado y futuro. Edmetic, v. 3, n. 1, p. 8-25, 2014.

Marta-Lazo, C.; Ortiz-Sobrino, M. A. Gestión de los fondos documentales en Radio Nacional de España. El Profesional de la Información, v. 22, n. 5, p. 399-403, 2013.

Marta-Lazo, C.; Segura, A. La radio educativa en la era del dial infinito. In: Gertrudix, M.; Gertrudix, F.; García, F. (Coord.). Actas II Congreso Internacional Sociedad Digital. Madrid: Icono 14, 2011. p. 341-355.

Martín-Pena, D.; Contreras, P.; Aguaded, J. I. Programación, publicidad y responsabilidad social en las radios universitarias españolas. Observatorio (OBS*), v. 9, n. 4, p. 141-157, 2015. http://doi.org/10.7458/obs942015879
Martín-Pena, D.; Contreras, P. Las radios universitarias en España: inicios, evolución y panorama actual. In: Martín-Pena, D.; Ortiz, M. A (Ed.). Radios Universitarias en América y Europa, Madrid: Fragua. 2014. p. 88-100.

Martín-Pena, D.; Parejo, M.; Vivas, A. La radio universitaria: gestión de la información, análisis y modelos de organización. Barcelona: Gedisa, 2016.

Ortiz-Sobrino, M. A. Radio y post-radio en España: una cohabitación necesaria y posible. Área Abierta, v. 12. n. 2, p. 1-16, 2012.

Ortiz-Sobrino, M. A. La radio española en el umbral de la web 3.0. In: Ortiz, M. A.; López Vidales, N. (Ed.). Radio 3.0: una nueva radio para una nueva era, la democratización de los contenidos. Madrid: Fragua. 2011. p. 41-66.

Ortiz-Sobrino, M. A; Peña, P. Radio Intercontinental-Radio Inter: 60 años de radio. Vivat Academia, v. 13, n. 113, p. 1-16, 2010.

Padilla, G.; Calvo, E. Radio 3.0. Éxito durmiente. In: Ortiz, M. A.; López Vidales, N. (Ed.). Radio 3.0: una nueva radio para una nueva era, la democratización de los contenidos. Madrid: Fragua. 2011. p. 281-300.

Pérez Alaejos, M. P.; López Merayo, A. Entre ondas y bits: el podcasting en las redes nacionales de radio españolas. Comunicaçao Midiática, v. 8, n. 3, p. 181-202, 2013.

Perona Páez, J. J. Edu-webs radiofónicas: experiencias españolas de educación en medios. Revista Comunicar, v. 27, n. 33, p. 107-114, 2009.

Piñeiro-Otero, T. De las ondas a los 140 caracteres. El uso de Twitter por los principales programas de la radio española. Palabra Clave, v. 18, n. 3, p. 815-841, 2015a.

Piñeiro-Otero,T.Información, multimedialidad y personalización en las radioapps de España y Portugal. El profesional de la Información, v. 24, n. 4, p. 463-471, 2015 b.

Piñeiro-Otero, T.; Videla Rodríguez, J. J. La participación de los oyentes en las radios APPs españolas: prácticas convencionales en la era de la portabilidad. AdComunica, Revista Estrategias, Tendencias e Innovación en Comunicación, n. 5, p. 67-89, 2013.

Ramos Ruiz, Á. Radio hertziana vs. Radio en Internet: un análisis comparativo. Opción, v. 31, n. 4, p. 757-774, 2015.

Rodríguez Pallarés, M.; Rodríguez Barba, D.; Peinado, F. Innovación en radio y nuevo modelo de negocio. El caso de Stream Radio. In: García Santamaría, J. V.; Pérez, F. (Coord.). Los medios digitales españoles: procesos de cambio e innovación. La Laguna, Tenerife: Latina, 2016. (Cuadernos Artesanos de Comunicación, CAC104).

Terol, R. Radio 3.0 en el entorno municipal: valores, herramientas y recursos, el caso de la emisora municipal Llosa FM. 2016. 326f. Tesis (Doctoral) - Universidad Politécnica de Valencia, Valencia, 2016.

Ventero, M.; Peña, P. Nuevas formas de participación en Radio: Radio tradicional y redes sociales. In: Ortiz, M. A.; López Vidales, N. (Ed.). Radio 3.0. Una nueva radio para una nueva era, la democratización de los contenidos. Madrid: Fragua. 2011. p. 231-247. 